HU-SEFT R 1995-18

\title{
Spinning Relativistic Particle in an External Electromagnetic Field
}

\author{
M. Chaichian ${ }^{a, b}$, R. Gonzalez Felipe ${ }^{b}$ and D. Louis Martinez ${ }^{c}$ \\ ${ }^{a}$ Laboratory for High Energy Physics, Department of Physics \\ ${ }^{b}$ Research Institute for High Energy Physics \\ P.O. Box 9 (Siltavuorenpenger $20 \mathrm{C}$ ) \\ FIN-00014 University of Helsinki, Finland \\ ${ }^{c}$ Department of Physics, University of Manitoba \\ Winnipeg MB R3T 2N2, Canada
}

\begin{abstract}
The Hamiltonian formulation of the motion of a spinning relativistic particle in an external electromagnetic field is considered. The approach is based on the introduction of new coordinates and their conjugated momenta to describe the spin degrees of freedom together with an appropriate set of constraints in the Dirac formulation. For particles with gyromagnetic ratio $g=2$, the equations of motion do not predict any deviation from the standard Lorentz force, while for $g \neq 2$ an additional force, which corresponds to the magnetic dipole force, is obtained.
\end{abstract}


The description of the motion of a spinning relativistic particle in $3+1$ dimensions has been a subject of research for many years. Equations describing the evolution of the classical spin in a uniform and static external electromagnetic field have been proposed by several authors [1, 2]. Modifications of the Lorentz force law for spinning particles moving in a slowly varying external electromagnetic field together with some generalizations for the equations describing the spin precession in this case have also been suggested [3]-[6]. Some Lagrangian and Hamiltonian formulations, which allow one to obtain the equations of motion from an action principle, have been proposed [7][13]. Most of them rely on the use of Grassmann variables [7]-[10]. In [11] the analogy with the relativistic quantum equations was used as a guiding principle. Recently, in Ref. [12] a canonical procedure has been proposed based on a Routhian function [14] from which the equations of motion for a spinning relativistic particle coupled to an external electromagnetic and a gravitational field can be derived. Also, in [13] a Lagrangian formulation based on classical commuting variables was proposed. It is however amusing that there is, even today, an ongoing debate as to the torque and force on such particles.

In this letter we shall consider the Hamiltonian formulation for the description of the motion of a spinning relativistic particle in $3+1$ space-time dimensions in an homogeneous constant electromagnetic field. The model is constructed by analogy with the one presented in [15] for anyons in $2+1$ dimensions. It uses the idea of expressing the antisymmetric spin tensor in terms of some new coordinates and their conjugated momenta as in the case of the orbital angular momentum tensor. We start with a Hamiltonian formulation of the problem by introducing the corresponding constraints in the theory. The Lagrangian can then be derived by using the usual Legendre transformation. We hope that the present model will provide an alternative formulation to the ones discussed in the literature and mentioned above.

Let the position of the relativistic particle be described by the four vector $x^{\mu}$ ( $\mu=0,1,2,3)$, and the spin of the particle by the antisymmetric spin matrix $S_{\mu \nu}$. We shall introduce the auxiliary coordinates $n^{\mu}$ to describe the spin. Therefore, in the phase space we work with the configuration space variables $x^{\mu}, n^{\mu}$ and their conjugated momenta $p_{\mu}, p_{\mu}^{(n)}$. We can write the following Poisson bracket (PB) relations:

$$
\begin{array}{r}
\left\{x_{\mu}, p_{\nu}\right\}=-g_{\mu \nu},\left\{x_{\mu}, x_{\nu}\right\}=\left\{p_{\mu}, p_{\nu}\right\}=0 \\
\left\{n_{\mu}, p_{\nu}^{(n)}\right\}=-g_{\mu \nu},\left\{n_{\mu}, n_{\nu}\right\}=\left\{p_{\mu}^{(n)}, p_{\nu}^{(n)}\right\}=0
\end{array}
$$

where the metric tensor $g_{\mu \nu}=\operatorname{diag}(1,-1,-1,-1)$. 
Let us define the antisymmetric spin matrix $S_{\mu \nu}$ as follows:

$$
S_{\mu \nu}=n_{\mu} p_{\nu}^{(n)}-n_{\nu} p_{\mu}^{(n)} .
$$

The total angular momentum matrix $M_{\mu \nu}$ can thus be written as

$$
M_{\mu \nu}=L_{\mu \nu}+S_{\mu \nu},
$$

where $L_{\mu \nu}=x_{\mu} p_{\nu}-x_{\nu} p_{\mu}$.

It is straightforward to verify that the four-vector $p_{\mu}$ and the tensor $M_{\mu \nu}$ satisfy the Poincaré algebra

$$
\begin{array}{r}
\left\{p_{\mu}, p_{\nu}\right\}=0, \\
\left\{M_{\mu \nu}, p_{\lambda}\right\}=-g_{\mu \lambda} p_{\nu}+g_{\nu \lambda} p_{\mu}, \\
\left\{M_{\mu \nu}, M_{\lambda \rho}\right\}=-g_{\mu \lambda} M_{\nu \rho}-g_{\nu \rho} M_{\mu \lambda}+g_{\mu \rho} M_{\nu \lambda}+g_{\nu \lambda} M_{\mu \rho} .
\end{array}
$$

Now we would like to consider the interaction of a spinning particle with gyromagnetic ratio $g=2$, electric charge $e$ and mass $m$ with a uniform static external electromagnetic field. A vector potential describing such a field can be expressed as

$$
A_{\mu}=-\frac{1}{2} F_{\mu \nu} x^{\nu},
$$

where $F_{\mu \nu}$ is the electromagnetic field tensor.

In order to describe the motion of such a particle we shall follow a Hamiltonian formulation analogous to the one presented in [15] and based on the imposed constraints and the algebraic properties of the system.

Let us consider the following extended (with respect to the Poincaré) algebra:

$$
\begin{array}{r}
\left\{\bar{\pi}_{\mu}, \bar{\pi}_{\nu}\right\}=e F_{\mu \nu}, \\
\left\{M_{\mu \nu}, \bar{\pi}_{\lambda}\right\}=-g_{\mu \lambda} \bar{\pi}_{\nu}+g_{\nu \lambda} \bar{\pi}_{\mu}, \\
\left\{M_{\mu \nu}, M_{\lambda \rho}\right\}=-g_{\mu \lambda} M_{\nu \rho}-g_{\nu \rho} M_{\mu \lambda}+g_{\mu \rho} M_{\nu \lambda}+g_{\nu \lambda} M_{\mu \rho}, \\
\left\{F_{\mu \nu}, \bar{\pi}_{\lambda}\right\}=0, \\
\left\{F_{\mu \nu}, F_{\lambda \rho}\right\}=0, \\
\left\{M_{\mu \nu}, F_{\lambda \rho}\right\}=-g_{\mu \lambda} F_{\nu \rho}-g_{\nu \rho} F_{\mu \lambda}+g_{\mu \rho} F_{\nu \lambda}+g_{\nu \lambda} F_{\mu \rho},
\end{array}
$$

where $\bar{\pi}_{\mu}=p_{\mu}+e A_{\mu}$.

It is not difficult to prove that the operator

$$
C=\bar{\pi}^{2}-e F_{\mu \nu} M^{\mu \nu}
$$


is the Casimir operator of the algebra (6). By using the relation

$$
F_{\mu \nu} L^{\mu \nu}=4(p A)
$$

Eq.(7) can be written in the form:

$$
C=\pi^{2}-e F_{\mu \nu} S^{\mu \nu}
$$

with $\pi_{\mu}=p_{\mu}-e A_{\mu}$. By fixing the value of $C$ to be equal to $m^{2}$ we can then take the quantity

$$
\Phi=\pi^{2}-e F_{\mu \nu} S^{\mu \nu}-m^{2}
$$

as a first-class constraint [16] in the Hamiltonian formulation.

Next we should require that in the rest frame of the particle the spin tensor $S_{\mu \nu}$ has only three independent components. This is usually done by imposing the conditions:

$$
S_{\mu \nu} \dot{x}^{\nu}=0
$$

where $\dot{x}^{\mu}$ is the four-velocity and the dot denotes the derivative with respect to the proper time $\tau$. Since we are considering the Hamiltonian formalism we should employ the momenta instead of the velocities. In an external electromagnetic field we thus expect the use of $\pi^{\mu}$ instead of $\dot{x}^{\mu}$. On the other hand, it is easy to see that from the definition of the spin tensor (2) it is sufficient to impose the constraints

$$
\left(\pi p^{(n)}\right)=0,(\pi n)=0,
$$

in order to guarantee that

$$
S_{\mu \nu} \pi^{\nu}=0 .
$$

Therefore, in our Hamiltonian formulation we shall consider the following set of primary constraints:

$$
\begin{array}{r}
\Phi=\pi^{2}-e F_{\mu \nu} S^{\mu \nu}-m^{2}, \\
\varphi_{1}=\left(\pi p^{(n)}\right), \\
\varphi_{2}=(\pi n) .
\end{array}
$$

Since

$$
\begin{array}{r}
\left\{\Phi, \varphi_{1}\right\}=0 \\
\left\{\Phi, \varphi_{2}\right\}=0 \\
\left\{\varphi_{1}, \varphi_{2}\right\}=\pi^{2}+\frac{e}{2} F_{\mu \nu} S^{\mu \nu}
\end{array}
$$


the constraint $\Phi$ is a first-class constraint, while $\varphi_{1}$ and $\varphi_{2}$ form a pair of second-class constraints. We shall define the total Hamiltonian of the system as

$$
H=\Lambda \Phi+\lambda_{1} \varphi_{1}+\lambda_{2} \varphi_{2}
$$

where $\Lambda, \lambda_{1}$ and $\lambda_{2}$ are Lagrange multipliers and the canonical Hamiltonian $H_{c a n}$ is taken to be equal to zero.

From the consistency conditions on the primary constraints (12), i.e. from the conditions

$$
\begin{aligned}
\dot{\Phi}=\{\Phi, H\} & =0, \\
\dot{\varphi}_{1}=\left\{\varphi_{1}, H\right\}=\lambda_{2}\left(\pi^{2}+\frac{e}{2} F_{\mu \nu} S^{\mu \nu}\right) & =0, \\
\dot{\varphi_{2}}=\left\{\varphi_{2}, H\right\}=-\lambda_{1}\left(\pi^{2}+\frac{e}{2} F_{\mu \nu} S^{\mu \nu}\right) & =0,
\end{aligned}
$$

it follows that

$$
\lambda_{1}=\lambda_{2}=0
$$

on the constrained surface and that no new constraints appear in the Dirac algorithm. The Lagrange multiplier $\Lambda$ remains undetermined.

Notice that the number of physical degrees of freedom in configuration space is equal to $8-1-2 / 2=6$. It is thus expected that 3 of these correspond to the position of the relativistic particle and the other 3 to the components of the spin.

It is useful to define the spin four-vector through the relation:

$$
S_{\mu}=\frac{1}{2} \varepsilon_{\mu \alpha \beta \gamma} \frac{\pi^{\alpha}}{\sqrt{\pi^{2}}} S^{\beta \gamma}
$$

which in the absence of an external electromagnetic field reduces to the usual PauliLubanski four-vector [17]. Using Eqs. (11) and (17) it is not difficult to prove that the spin matrix $S_{\mu \nu}$ can be written on the constrained surface as follows:

$$
S_{\mu \nu}=\varepsilon_{\mu \nu \alpha \beta} S^{\alpha} \frac{\pi^{\beta}}{\sqrt{\pi^{2}}}
$$

It also follows that

$$
S^{2} \equiv S_{\mu} S^{\mu}=-\frac{1}{2} S_{\mu \nu} S^{\mu \nu}
$$


The Dirac-Hamilton equations of motion which follow from the PB (11) and the Hamiltonian (14) read

$$
\begin{array}{r}
\dot{x}^{\mu}=2 \Lambda \pi^{\mu}, \\
\dot{n}^{\mu}=2 \Lambda e F^{\mu \nu} n_{\nu}, \\
\dot{p}_{\mu}=\Lambda e F_{\mu \nu} \pi^{\nu}, \\
\dot{p}_{\mu}^{(n)}=2 \Lambda e F_{\mu \nu} p^{(n) \nu},
\end{array}
$$

and they should hold together with the constraints (12).

Let us now rewrite these equations in a more familiar way. First notice that

$$
\dot{\pi}_{\mu}=\dot{p}_{\mu}+\frac{e}{2} F_{\mu \nu} \dot{x}^{\nu} .
$$

Using Eqs.(20) and choosing $\Lambda=1 / 2 m$ we obtain

$$
\ddot{x}_{\mu}=\frac{e}{m} F_{\mu \nu} \dot{x}^{\nu} .
$$

Also from the definition of the spin matrix (cf. Eq.(国)) and the equations of motion (20) it follows that

$$
\dot{S}_{\mu \nu}=-\frac{e}{m} F^{\alpha \beta}\left(S_{\mu \alpha} g_{\nu \beta}+S_{\nu \beta} g_{\mu \alpha}\right) .
$$

Eqs.(21) and (22) imply that the Frenkel conditions (10) are preserved in proper time, i.e.

$$
\frac{d}{d \tau}\left(S_{\mu \nu} \dot{x}^{\nu}\right)=0 .
$$

Also from (19) and (22) it follows that the value of the spin is a constant of motion:

$$
\frac{d}{d \tau}\left(S^{2}\right)=0 .
$$

Finally, Eq.(22) can be rewritten in terms of the spin vector defined in (17). We have

$$
\dot{S}_{\mu}=\frac{e}{m} F_{\mu \nu} S^{\nu},
$$

which coincides with the Bargmann-Michel-Telegdi equation [2] for spinning particles with $g=2$.

For completeness, let us derive the Lagrange function corresponding to the total Hamiltonian (14). The derivation is very similar to the one presented in [15]. The first 
step is to express the Lagrange multipliers $\Lambda, \lambda_{1}$ and $\lambda_{2}$ in terms of the generalized coordinates and velocities. This can be achieved by using the relations

$$
\begin{gathered}
\dot{x}_{\mu}=2 \Lambda \pi_{\mu}+\lambda_{1} p_{\mu}^{(n)}+\lambda_{2} n_{\mu} \\
\dot{n}_{\mu}=2 \Lambda e F_{\mu \nu} n^{\nu}+\lambda_{1} \pi_{\mu},
\end{gathered}
$$

and the constraints (12).

The Lagrange function is equal to

$$
L=(\dot{x} p)+\left(\dot{n} p^{(n)}\right)=(\dot{x} \pi)+\left(\dot{n} p^{(n)}\right)+e(A \dot{x})
$$

where the momenta $\pi_{\mu}$ and $p_{\mu}^{(n)}$ should be expressed in terms of the generalized coordinates and velocities using Eqs.(26). Since

$$
\begin{array}{r}
(\dot{x} \pi)=2 \Lambda \pi^{2}=2 \Lambda\left(e F_{\mu \nu} S^{\mu \nu}+m^{2}\right), \\
\left(\dot{n} p^{(n)}\right)=2 \Lambda e F_{\mu \nu} n^{\nu} p^{(n) \mu}=-\Lambda e F_{\mu \nu} S^{\mu \nu},
\end{array}
$$

then we have

$$
L=2 m^{2} \Lambda\left(1+\frac{e}{2 m^{2}} F_{\mu \nu} S^{\mu \nu}\right)+e(A \dot{x})
$$

Up to terms linear in the electromagnetic field strength we find from Eqs.(26) and (12):

$$
\Lambda=\frac{(\dot{n} \dot{x})}{2 m \sqrt{\dot{n}^{2}}}+O\left(e^{2} F^{2}\right)
$$

and thus we obtain finally

$$
L=\frac{m(\dot{n} \dot{x})}{\sqrt{\dot{n}^{2}}}\left(1+\frac{e}{2 m^{2}} F_{\mu \nu} S^{\mu \nu}\right)+e(A \dot{x})+O\left(e^{2} F^{2}\right)
$$

where

$$
S^{\mu \nu}=\frac{m}{\sqrt{\dot{n}^{2}}}\left(n^{\mu} \dot{x}^{\nu}-n^{\nu} \dot{x}^{\mu}\right)-\frac{m(\dot{n} \dot{x})}{\left(\dot{n}^{2}\right)^{3 / 2}}\left(n^{\mu} \dot{n}^{\nu}-n^{\nu} \dot{n}^{\mu}\right)
$$

It is worth mentioning that in the absence of an external electromagnetic field from the equations of motion (20) it follows that $\dot{n}^{\mu}=0$. In this case it is more convenient to adopt a first order formalism (e.g. as advocated in [18, 19]) and take the Lagrangian as

$$
L=(\dot{x} p)+\left(\dot{n} p^{(n)}\right)-\Lambda\left(p^{2}-m^{2}\right)-\lambda_{1}\left(p p^{(n)}\right)-\lambda_{2}(p n) .
$$


We have presented the Hamiltonian description for the motion of a particle with gyromagnetic ratio $g=2$ in an external electromagnetic field. The main ingredients of the model are the following: the phase space of the system is given by the coordinates $x^{\mu}$ and $n^{\mu}$ and their conjugated momenta $p_{\mu}$ and $p_{\mu}^{(n)}$ respectively; the antisymmetric spin matrix $S_{\mu \nu}$ is defined in terms of the four vectors $n_{\mu}$ and $p_{\mu}^{(n)}$ (see Eq.(2)) by analogy with the orbital angular momentum tensor; the total Hamiltonian is given in (14) with the primary constraints defined in (12). Out of these starting points we have obtained the equations of motion (21) and (22) (or equivalently Eq. (251).

Let us now consider the case of a particle with gyromagnetic ratio $g \neq 2$. A straightforward generalization of our previous $(g=2)$ analysis to the case $g \neq 2$ would be the introduction of the factor $g / 2$ in the interaction term $e F_{\mu \nu} S^{\mu \nu}$ of the constraint $\Phi$ in Eqs.(12). However, in order to keep the latter constraint as first-class and the constraints $\varphi_{1}$ and $\varphi_{2}$ as second-class, we should further modify the constraint $\Phi$. Taking the linear combination

$$
\tilde{\Phi}=\pi^{2}-\frac{e g}{2} F_{\mu \nu} S^{\mu \nu}-m^{2}+A \varphi_{1}+B \varphi_{2},
$$

and imposing the conditions $\left\{\tilde{\Phi}, \varphi_{1}\right\}=\left\{\tilde{\Phi}, \varphi_{2}\right\}=0$ for the constraint $\tilde{\Phi}$ to be firstclass, we can determine the coefficients $A$ and $B$. Finally we find

$$
\tilde{\Phi}=\pi^{2}-\frac{e g}{2} F_{\mu \nu} S^{\mu \nu}-m^{2}-\frac{e(g-2)}{\left(\pi^{2}+\frac{e}{2} F_{\mu \nu} S^{\mu \nu}\right)} F^{\alpha \beta} \pi_{\alpha} S_{\beta \gamma} \pi^{\gamma}
$$

The equations of motion which follow from the new Hamiltonian

$$
H=\Lambda \tilde{\Phi}+\lambda_{1} \varphi_{1}+\lambda_{2} \varphi_{2}
$$

(with $\varphi_{1}$ and $\varphi_{2}$ defined in (12)) read as follows:

$$
\begin{array}{r}
\dot{x}^{\mu}=2 \Lambda \pi^{\mu}-\frac{\Lambda e(g-2)}{\left(\pi^{2}+\frac{e}{2} F_{\mu \nu} S^{\mu \nu}\right)} F_{\alpha \beta} \pi^{\alpha} S^{\beta \mu}, \\
\dot{n}^{\mu}=\Lambda e g F^{\mu \nu} n_{\nu}-\frac{\Lambda e(g-2)}{\left(\pi^{2}+\frac{e}{2} F_{\mu \nu} S^{\mu \nu}\right)} F^{\alpha \beta} \pi_{\alpha} n_{\beta} \pi^{\mu}, \\
\dot{\pi}_{\mu}=2 \Lambda e F_{\mu \nu} \pi^{\nu}-\frac{\Lambda e^{2}(g-2)}{\left(\pi^{2}+\frac{e}{2} F_{\mu \nu} S^{\mu \nu}\right)} F_{\alpha \beta} \pi^{\alpha} F_{\mu \nu} S^{\beta \nu}, \\
\dot{p}_{\mu}^{(n)}=\Lambda e g F_{\mu \nu} p^{(n) \nu}-\frac{\Lambda e(g-2)}{\left(\pi^{2}+\frac{e}{2} F_{\mu \nu} S^{\mu \nu}\right)} F^{\alpha \beta} \pi_{\alpha} p_{\beta}^{(n)} \pi_{\mu} .
\end{array}
$$


For the spin tensor $S_{\mu \nu}$ and vector $S_{\mu}$ we get in turn:

$$
\begin{aligned}
\dot{S}_{\mu \nu}= & -\Lambda e g F^{\alpha \beta}\left(S_{\mu \alpha} g_{\nu \beta}+S_{\nu \beta} g_{\mu \alpha}\right) \\
& +\frac{\Lambda e(g-2)}{\left(\pi^{2}+\frac{e}{2} F_{\mu \nu} S^{\mu \nu}\right)} \pi_{\alpha} F^{\alpha \beta}\left(S_{\beta \mu} \pi_{\nu}+S_{\nu \beta} \pi_{\mu}\right), \\
\dot{S}_{\mu}= & \Lambda e g F_{\mu \nu} S^{\nu}-\frac{\Lambda e(g-2)}{\pi^{2}} \pi_{\mu} F_{\alpha \beta} \pi^{\alpha} S^{\beta} \\
& -\frac{\Lambda e^{2}(g-2)}{\left(\pi^{2}\right)^{3 / 2}\left(\pi^{2}+\frac{e}{2} F_{\mu \nu} S^{\mu \nu}\right)} \pi_{\mu} \varepsilon_{\alpha \beta \gamma \delta} F^{\alpha \alpha^{\prime}} S_{\alpha^{\prime}} F^{\beta \beta^{\prime}} \pi_{\beta^{\prime}} S^{\gamma} \pi^{\delta} .
\end{aligned}
$$

We note that from the equations of motion (34)-(35) it follows that the value of the spin (19) is preserved in time. Moreover, using the expression for $\pi^{\mu}$ which follows from the first equation in (34), i.e.

$$
\pi^{\mu}=\frac{1}{2 \Lambda}\left(\dot{x}^{\mu}+\frac{\Lambda e(g-2)}{\left(\pi^{2}+\frac{e}{2} F_{\mu \nu} S^{\mu \nu}\right)} F_{\alpha \beta} \pi^{\alpha} S^{\beta \mu}\right),
$$

and substituting it into the definition of the spin vector (17), it is easy to show that

$$
S_{\mu}=\frac{1}{4 \Lambda \sqrt{\pi^{2}}} \varepsilon_{\mu \alpha \beta \gamma} \dot{x}^{\alpha} S^{\beta \gamma}
$$

Thus the condition $S_{\mu} \dot{x}^{\mu}=0$ is automatically satisfied by the equations of motion.

For the case $g=2$, if one chooses $\Lambda=1 / 2 m$, Eqs.(34)-(35) coincide with Eqs.(21) and (25). However, when $g \neq 2$, additional quadratic and higher order terms in the field strength $F_{\mu \nu}$ appear. These terms reproduce the Bargmann-Michel-Telegdi equation [2] in the linear approximation. Indeed, from (34)-(35) we obtain

$$
\begin{array}{r}
\ddot{x}_{\mu}=\frac{e}{m} F_{\mu \nu} \dot{x}^{\nu}+(g-2) O\left(e^{2} F^{2}\right), \\
\dot{S}_{\mu}=\frac{e g}{2 m} F_{\mu \nu} S^{\nu}-\frac{e(g-2)}{2 m} \dot{x}_{\mu} F_{\alpha \beta} \dot{x}^{\alpha} S^{\beta}+(g-2) O\left(e^{2} F^{2}\right) .
\end{array}
$$

We remark that the extra terms of order $e^{2} F^{2}$ in Eqs. 37) do not coincide with the ones previously obtained in the literature [5, 12, 13. One way to see that is to consider the nonrelativistic limit. For particles with $g=2$ our equations do not predict any deviation from the standard Lorentz force, while in Refs. [5, 12, 13] a term proportional to $g \vec{B} \times(\vec{S} \times \vec{E})$ is obtained. For $g \neq 2$, however, Eqs.(34) do predict in the nonrelativistic limit an additional force, namely,

$$
-\frac{e^{2}(g-2)}{2 m^{3}} \vec{B} \times(\vec{S} \times \vec{E})
$$

which modifies the Lorentz force.

We have considered so far the case when the electromagnetic field strength $F_{\mu \nu}$ is constant. We can modify the model to obtain the equations of motion in slowly 
varying fields. In the latter case, the constraint $\Phi$ defined in Eq.(9) as well as $\tilde{\Phi}$ in Eq.(32) will also generate the correct equations of motion so that in the nonrelativistic limit we obtain the term $\frac{e g}{2 m} \vec{\nabla}(\vec{B} \cdot \vec{S})$ [5, 12, 13].

We are grateful to M. Bander, I. Khriplovich and G. Kunstatter for useful discussions.

\section{References}

[1] J. Frenkel, Z. Phys. 37 (1926) 243.

[2] V. Bargmann, L. Michel and V.L. Telegdi, Phys. Rev. Lett. 2 (1959) 435.

[3] Y. Aharonov and A. Casher, Phys. Rev. Lett. 53 (1984) 319.

[4] L. Vaidman, Am. J. Phys. 58 (1986) 978.

[5] J. Anandan, Phys. Lett. A138 (1989) 347.

[6] J.W. van Holten, Nucl. Phys. B356 (1991) 3.

[7] A. Barducci, R. Casalbouni and L. Lusanna, Nuovo Cimento A35 (1976) 389.

[8] F. Ravndal, Phys. Rev. D21 (1980) 2823.

[9] P.L. Nash, J. Math. Phys. 25 (1984) 2104.

[10] R.H. Rietdijk and J.W. van Holten, Class. Quantum Grav. 10 (1992) 575.

[11] I.B. Khriplovich, Zh. Eksp. Teor. Fiz. 96 (1989) 385 [Sov. Phys. JETP 69 (1989) 217].

[12] K. Yee and M. Bander, Phys. Rev. D48 (1993) 2797.

[13] J.P. Costella and B.H.J. McKellar, Int. J. Mod. Phys. A9 (1994) 461.

[14] L.D. Landau and E.M. Lifshitz, Classical Mechanics (Pergamon Press, Oxford, 1960).

[15] M. Chaichian, R. Gonzalez Felipe and D. Louis Martinez, Phys. Rev. Lett. 71 (1993) 3405; Phys. Rev. Lett. 73 (1994) 2009. 
[16] P.A.M. Dirac, Lectures in Quantum Mechanics (Belfer Graduate School of Science, Yeshiva University, New York, 1964).

[17] See for example, C. Itzykson and J.-B. Zuber, Quantum Field Theory (McGraw Hill, New York, 1980).

[18] L. Faddeev and R. Jackiw, Phys. Rev. Lett. 60 (1988) 1692.

[19] R. Jackiw and V.P. Nair, Phys. Rev. Lett. 73 (1994) 2007. 